\title{
A Simple And Efficient Design Of Instantaneous Frequency Measurement Encoder With Phase Comparison Method
}

\author{
Feng Zhang ${ }^{1,2}$, Peng He ${ }^{1,2}$, Anhui Liang ${ }^{1,2}$ \\ 1. The 41 st Research Institute of CETC \\ 2. Science and Technology on Electronic Test \& Measurement Laboratory \\ Qingdao, China \\ zhangfeng880523@163.com,xibeilanghp@163.com,lahidea@126.com
}

Keywords: instantaneous frequency measurement; phase comparison method; encoder

\begin{abstract}
This paper presents a simple and efficient design of instantaneous frequency measurement encoder with phase comparison method. Firstly, in order to improve the accuracy of the sampling data, the software filter module is added in the encoder. Secondly, the encoder uses hierarchical lookup table to reduce the complexity of encoder design. Finally, the independent programming makes the encoder have good expansibility and portability. In practical application, the encoder has achieved good effect and has been widely used in frequency measurement receivers.
\end{abstract}

\section{Introduction}

Carrier frequency is one of the key characteristic parameters in weapon equipment, and frequency measuring receiver is widely used in weapon equipment ${ }^{[1,2]}$. The instantaneous frequency measurement with phase comparison method is a common frequency measurement method in frequency measurement receiver ${ }^{[3,4]}$. In the instantaneous frequency measurement with phase comparison method, the signal phase information is converted into phase data by using signal phase autocorrelation technique. Through the calculation and processing of phase data, the frequency measurement process is finally realized. The instantaneous frequency measurement module is mainly composed of limiting amplifier, phase detector and encoder ${ }^{[5,6]}$. The main workflow of traditional encoder is shown in Figure 1.

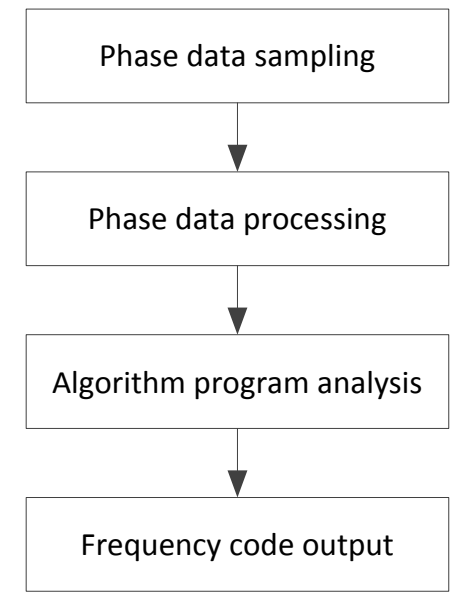

Figure 1: Traditional encoder workflow

As shown above, the workflow of traditional encoder mainly includes four units, the main functions of each unit are as follows ${ }^{[7,8,9]}$.

(1) Phase data sampling: Sampling phase data of frequency points to be measured.

(2) Phase data processing: Using CORDIC algorithm to deal with the phase data.

(3) Algorithm program analysis: By analyzing the processed phase data and combining the phase data of all measurable frequency points in the program, the corresponding frequency coding is obtained and optimized. 
(4) Frequency code output: The frequency code obtained by the program analysis is output as the final processing result of the encoder.

Although the traditional encoder can realize the main function of instantaneous frequency measurement, it has the following deficiencies.

(1) There is a large error in the sampling process of the measured frequency phase data.

(2) The CORDIC algorithm in phase data processing increases the complexity of encoder design.

(3) The algorithm program cannot be reused between different frequency measurement modules, and it is not scalable and portable.

In order to improve the deficiencies of traditional encoder design, this paper presents a simple and efficient design of instantaneous frequency measurement encoder with phase comparison method. This method improves the sampling data accuracy, reduce the complexity of the encoder design. Moreover, the algorithm program designed by this method has good expansibility and portability.

\section{Encoder design}

The new encoder workflow of this paper is shown in Figure 2.

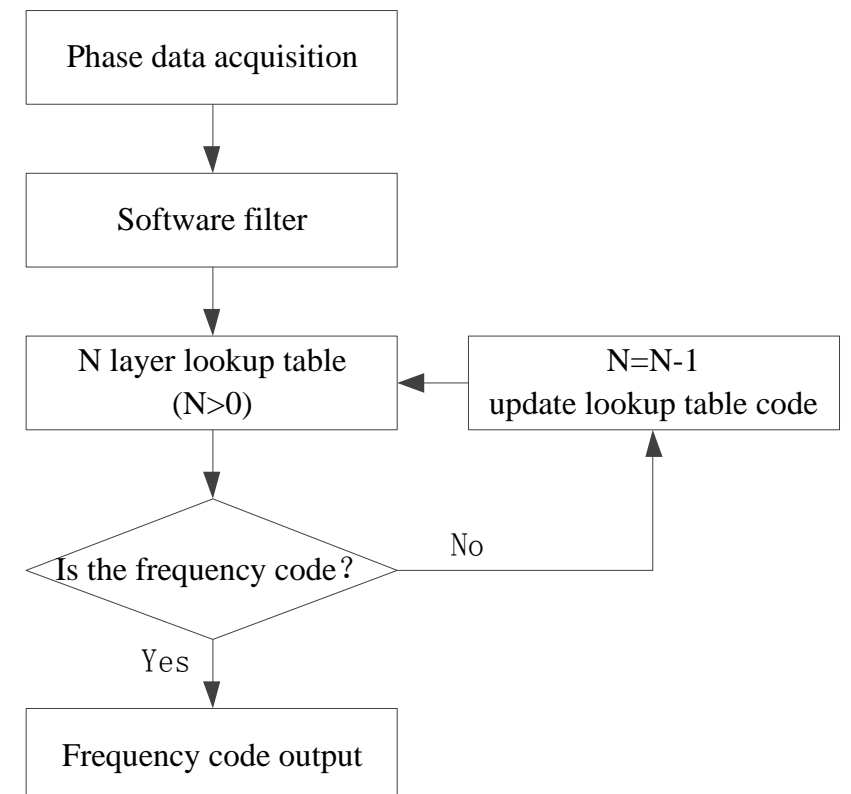

Figure 2: New encoder workflow

As shown above, the workflow of new encoder mainly includes four units, the main functions of each unit are as follows.
(1) Phase data acquisition: Collecting phase data of the frequency point to be measured.

(2) Software filter: Optimizing the phase data acquired.

(3) Hierarchical lookup table: Using the optimized phase data to search the table and retrieve the corresponding frequency coding.

(4) Frequency coding output: The retrieved frequency code is output as the final processing result of encoder.

The instantaneous frequency measurement module usually uses several sets of phase detector to improve the range and precision of frequency measurement. By designing the delay line length, the encoder can obtain several sets of phase data with different periods. The method proposed in this paper is based on two sets of phase data with different periods. This paper will describe the new encoder design from three aspects: software filter, hierarchical table lookup and independent programming.

\subsection{Software filter}

The phase data acquired by encoder is the key input parameter of the encoder. The accuracy of the data will directly affect the final measurement results. The new encoder adds software filtering module to improve the accuracy of phase data acquisition. The software filter module includes two units: mean filter and module value calculation.

Firstly, the mean filter is used to do the mean filtering for the sampled phase data. It will reduce the impact of noise signal on the sampling error, and further improve the accuracy of phase data. Taking the phase data of two groups of frequency measurement as an example, the data curves before and after using the software filter are shown in Figure 3 and Figure 4.

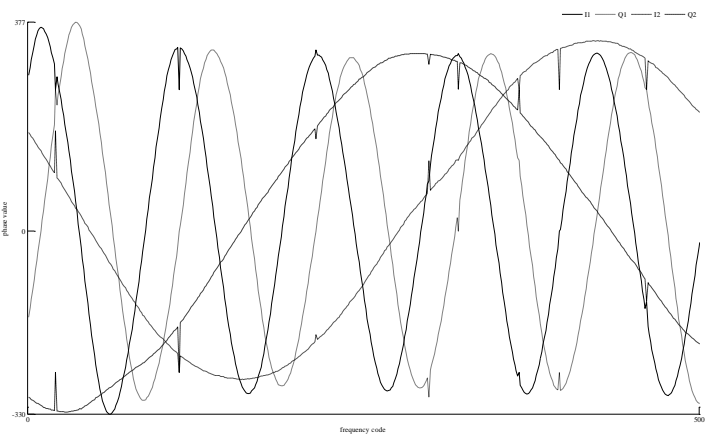

Figure 3: Phase data before using software filter 


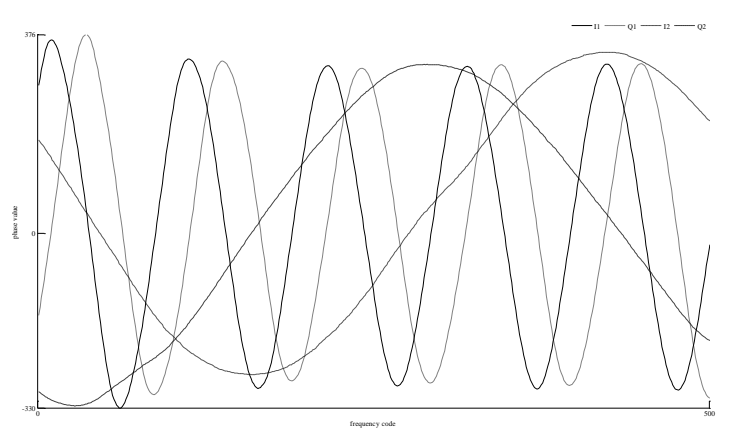

Figure 4: Phase data using software filter

By comparing Figure 3 and Figure 4, it is found that the abrupt error points are significantly reduced in the phase data after software filter. However, the amplitude range of different phase data curves is inconsistent, which is not conducive to subsequent processing. Next, the encoder will unify the amplitude range of the phase data curve by the calculation of module values. The specific equation are shown below.

$$
\begin{aligned}
M_{I} & =\frac{K_{I}\left(I-R_{I}\right)}{\sqrt{\left(I-R_{I}\right)^{2}+\left(Q-R_{Q}\right)^{2}}} \\
M_{Q} & =\frac{K_{Q}\left(Q-R_{Q}\right)}{\sqrt{\left(Q-R_{Q}\right)^{2}+\left(I-R_{I}\right)^{2}}}
\end{aligned}
$$

Such as the equation (1) and the equation (2), I and Q are two orthogonal phase data obtained after mean filter. $R_{I}$ and $\mathrm{R}_{\mathrm{Q}}$ are reference values for module value calculation. $\mathrm{K}_{\mathrm{I}}$ and $\mathrm{K}_{\mathrm{Q}}$ are scaling factors for module value calculation. $\mathrm{M}_{\mathrm{I}}$ and $\mathrm{M}_{\mathrm{Q}}$ are the result of module value calculation. After calculating the phase data in Figure 4, the new phase data obtained within the measured frequency range is shown in Figure 5.

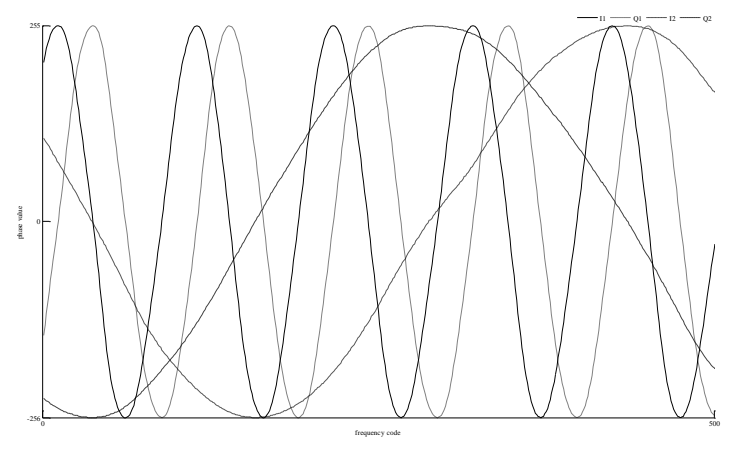

Figure 5: New phase data after module value calculation
By comparing Figure 4 and Figure 5, it is found that the amplitude range of the different phase data curves is consistent after the module value calculation. As shown in Figure 5, the encoder obtains two sets of orthogonal phase data over the entire frequency range: I1/Q1 and I2/Q2.

\subsection{Hierarchical table lookup}

Compared with the ordinary look-up table operation, hierarchical lookup table is to complete the table lookup process by using many different levels of look-up table index. The first step, the hierarchical table lookup will use I2 and Q2 data for the first level of look-up table operations to get I1 and Q1 retrieval interval. The second step, the hierarchical table lookup will use I1 and Q1 data for second levels of look-up table operations to get the final frequency coding. I1 and Q1 retrieval interval is shown in Figure 6.

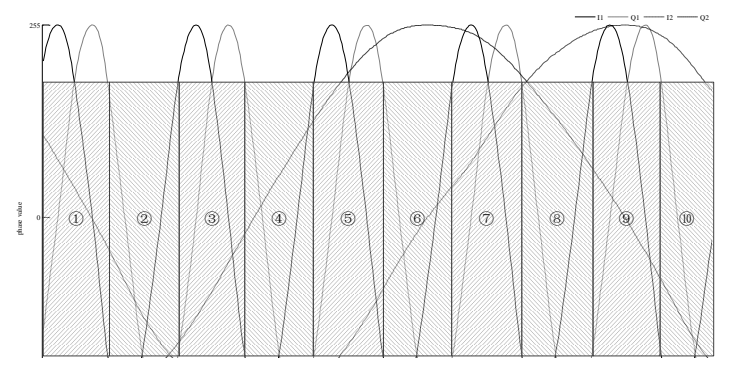

Figure 6: Retrieval interval

The density of curve data which located near the peak and trough is too high, and it is not conducive to the division of retrieval interval. Therefore, between the peak and trough of a higher degree of discrete data is selected as the retrieval interval division basis. Refer to the periodicity of all data changes, I2 and Q2 can divide I1 and Q1 into 10 different retrieval intervals. In each independent retrieval interval, the frequency code corresponds to the unique I1 (or Q1) data.

\subsection{Independent programming}

In the design of encoder, independent programming is to design the program and preset data separately. Different phase comparison instantaneous frequency measurement modules will use the same algorithm program, and all the preset data will be stored in the independent external data storage chip. In addition, the encoder design has preset data 
chip read and write function, and can achieve the external storage data real-time read and write.

When the encoder needs to retrieve the preset data, the encoder will read the data stored in the data storage chip, and then retrieve the operation to obtain the target data. The encoder can also update external storage preset data by writing data to the chip.

\section{Application and analysis}

In practical frequency measurement applications, the new encoder in this paper completes the frequency measurement of $-60 \mathrm{dBm}$ power, $1.3 \mathrm{GHz}$ bandwidth and $3 \mathrm{MHz}$ frequency step at room temperature. The final measurement error is shown in Figure 7.

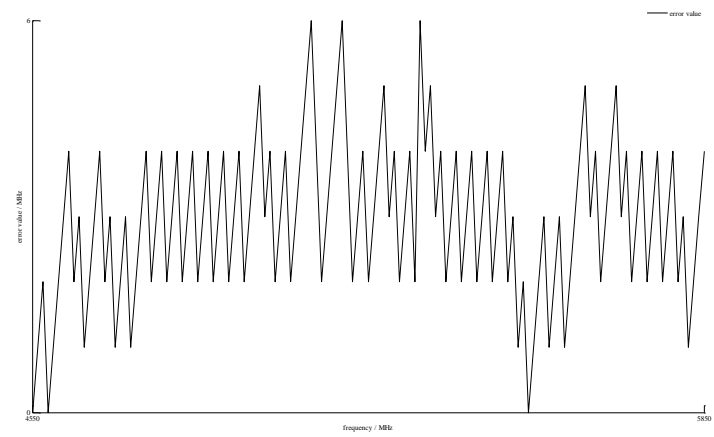

Figure 7: Measurement error

As shown above, the measurement error of all frequency points within the frequency range is within $6 \mathrm{MHz}$

\section{Conclusions}

In this paper, the simple and efficient design of instantaneous frequency measurement encoder with phase comparison method optimizes the traditional encoder, improves the sampling data accuracy, reduces the complexity of the encoder design, and realizes the scalability and portability of the encoder design. In practical application, the encoder realized by this design has achieved good frequency measurement effect and has been widely used in frequency measurement receivers.

\section{References}

[1]Burns K., "Tracking trends in military IMFs and DFDs", Microwave \& RF, 6, pp.163-171, (2009).
[2]Coupez J.P., Gruchala H., Slowik A., Recko C., "High Resolution IFMs", Microwaves Radar and Wireless Communications, 2, pp.484-487, (2002).

[3]Li Binyu, Xu Jiejing, Wei Bo, "Implementation of instantaneous frequency measurement based on phase difference by FPGA", Modern Electronics Technique, 36, pp.118-120, (2013).

[4]Sullivan W.B., "Instantaneous Frequency Measurement Receivers for Maritime Patrol", The Journal of electronic defense, 10, pp.21-22, (2002).

[5]Tatu S.O., “Ka-Band Direct Digital Receiver”, IEEE Trans. On Microwave Theory and Techniques, 50, pp.2436-2442, (2002).

[6]Wang Chunlei, Zhang Xuecheng, "Design of IFM Circuit Based on New Wide-band Phase Discriminator", Shipboard Electronic Countermeasure, 36, pp.106-108, (2013).

[7]Wang Yasen, Bao Qinglong, Cao Wushen, Liu Peng, Chen Zengping, "Research on a Digital Channelized IFM Receiver”, Radar Science and Technology, 5, pp.539-543, (2012)

[8]Xue Mingjun, Kong Xianhui, Zhang Hongmei, “An Engineering Realization of an Instantaneous Frequency Measurement Receiver by the Phase Comparison Method", Electronic Sci. \& Tech., 27, pp.103-105, (2014).

[9]Zhao Peng, Wang Zhiqiang, Fan Hailing, "Design of the Universal Phase-Detector IFM Model", Semiconductor Testing and Equipments, 3, pp.231-234, (2013). 